ARTIGO ORIGINAL ORIGINAL ARTICLE

\section{Análise de custo-efetividade da abiraterona para câncer de próstata metastático resistente à castração em pacientes com uso prévio de quimioterapia}

\author{
Cost-effectiveness analysis of abiraterone for metastatic, castrate- \\ resistant prostate cancer following previous chemotherapy
}

Lisiane Freitas Leal' ${ }^{1}$, Rafael José Vargas Alves ${ }^{1}$,Carisi Anne Polanczyk ${ }^{1,2}$, Rodrigo Antonini Ribeiro ${ }^{1,3}$

DOI: $10.21115 / J B E S . v 10 . n 2 . p 126-133$

\begin{abstract}
RESUMO
Objetivo: Avaliar a custo-utilidade e o impacto orçamentário $(\mathrm{IO})$ da abiraterona para o tratamento de câncer de próstata resistente à castração, em pacientes previamente tratados com docetaxel. Métodos: Foi construído um modelo de Markov com ciclos mensais sob a perspectiva do Sistema Único de Saúde (SUS), em um horizonte temporal de cinco anos e taxa de desconto de 5\%. A estimativa de efetividade foi oriunda do principal ensaio clínico dessa condição de saúde. Para dados de utilidade, aplicaram-se estimativas internacionais, enquanto para custos se utilizaram tabelas de remuneração do SUS. Para o IO, a população-alvo foi estimada com base em dados do Departamento de Informática do SUS (DATASUS). Resultados: A abiraterona ocasionou ganho de 1,045 ano de vida ajustado para qualidade (QALY) e 1,609 ano de vida ganho (AVG), enquanto para o placebo esses valores foram de 0,763 e 1,299, respectivamente. O custo total na estratégia abiraterona foi de $\mathrm{R} \$ 83.295$ e para o placebo, de R\$2.895. A relação de custo-efetividade incremental (RCEI) foi de R\$ 284.416 por QALY ganho. Em nenhuma das análises de sensibilidade os valores ficaram abaixo de R\$ 100.000 por QALY. Mesmo quando variados simultaneamente seis parâmetros, todos no seu limite mais favorável à abiraterona, os resultados seguiram elevados, com RCEI de R\$98.330 por QALY. O IO foi de R\$270 milhões em cinco anos no cenário mais conservador (tempo médio de tratamento de 7,4 meses e 10\% de novos casos/ano). Conclusão: A abiraterona se mostrou pouco custo-efetiva nesta situação clínica, com RCEI superior a nove vezes o PIB per capita por QALY, sendo os resultados robustos em análise de sensibilidade.
\end{abstract}

Keywords:

cost-benefit analysis, abiraterone acetate, Markov model

\begin{abstract}
Objective: To evaluate the cost-utility and the budget impact (BI) of abiraterone in patients with castration-resistant prostate cancer previously treated with docetaxel. Methods: A Markov model was constructed, with monthly cycles, under the perspective of the Brazilian Public Healthcare System (SUS), in a 5-year time horizon, and with a 5\% discount rate. The effectiveness estimate was obtained from the pivotal clinical trial for abiraterone in this health condition. For utility data, international estimates were applied; while for costs, SUS reimbursement information were used. In the $\mathrm{BI}$ analysis, the target population was estimated with claims data from DATASUS. Results: Abiraterone resulted in a gain of 1.045 quality-adjusted life years (QALY) and 1.609 life years gained (LYG), while for placebo these values were 0.763 and 1.299, respectively. The total cost for the abiraterone strategy was BRL 83,295, and for placebo, BRL 2,895. The incremental cost-effectiveness ratio (ICER)
\end{abstract}


was BRL 284,416 per QALY gained. None of the estimates from the sensitivity analysis was below BRL 100,000 per QALY. Even when six parameters were variated simultaneously in the range more favorable to abiraterone, the results were still elevated, with an ICER of BRL 98,330 per QALY. The BI was BRL 270 million in 5 years in the most conservative scenario (average time of the treatment of 7.4 months and $10 \%$ of the new cases/year). Conclusion: Abiraterone shows unfavorable cost-effective results for this clinical condition in Brazil, with an ICER above 9 times the per capita per QALY. Results were robust in sensitivity analysis.

\section{Introdução}

O câncer de próstata (CaP), sem considerar os tumores de pele não melanoma, é a neoplasia mais prevalente entre a população mundial masculina. No Brasil, estimam-se 68.220 casos novos de CaP para cada ano do biênio 2018-2019 (INCA, 2017), sendo a segunda causa de morte por neoplasias entre homens (INCA, 2014). Em geral, o CaP apresenta um comportamento indolente, beneficiando os pacientes com altas taxas de sobrevida em dez anos (Siegel et al., 2013). Entretanto, 10\% a 20\% desses casos se apresentam com metástases ao diagnóstico (CaPm), configurando um caráter mais agressivo à neoplasia. Para essa condição, o bloqueio androgênico (castração) é o tratamento de escolha, considerando-se os pacientes diagnosticados com doença localmente avançada ou metastática (Hellerstedt \& Pienta, 2002).

Apesar da efetividade do bloqueio hormonal no CaP, a maioria dos pacientes poderá desenvolver resistência à castração estabelecida por níveis de testosterona inferiores a $50 \mathrm{ng} / \mathrm{dl}$ (Cornford et al., 2017) associados à progressão de doença bioquímica (PSA) ou radiológica. O quimioterápico docetaxel foi o primeiro a demonstrar aumento de sobrevida global nessa condição (Tannock et al., 2004).

Pacientes que apresentam progressão do CaPm, mesmo tratados com docetaxel, podem ser beneficiados com terapia de segunda linha, estando indicado o medicamento cabazitaxel (de Bono et al., 2010). Esses dois regimes de quimioterapia em primeira e segunda linhas, docetaxel e cabazitaxel, respectivamente, foram responsáveis pela mudança no panorama dos pacientes com CaPm - não só pelo aumento de sobrevida global que demonstraram, mas também pelo fato de que por mais de meio século a hormonoterapia ser a única opção de tratamento para esses pacientes (Hellerstedt \& Pienta, 2002).

Nos últimos anos, além desses medicamentos quimioterápicos, foram introduzidas outras classes de fármacos para o tratamento de segunda linha (pós-docetaxel) do CaPm resistente à castração, como a abiraterona e a enzalutamida (Fizazi et al., 2012; Scher et al., 2012). O acetato de abiraterona, a partir do estudo COU-AA-301, demonstrou um aumento de sobrevida global em pacientes com CaPm resistente à castração após progressão com docetaxel. Nesse estudo, o braço do tratamento apresentou ganho na sobrevida global (razão de riscos = 0,65; IC95\%: 0,54-0,77; $\mathrm{p}<0,001$ ) com uma mediana de 14,8 meses, quando comparado com 10,9 meses do grupo placebo (De Bono et al., 2011). Além disso, esse medicamento tem apresentado benefícios clínicos para outros subgrupos de pacientes com essa condição (Logothetis et al., 2012; Van Praet et al., 2016).

No Brasil, desde o seu registro, o acetato de abiraterona vem sendo amplamente indicado e, em 2015, representou um custo superior a R\$ 5 milhões de reais ao Sistema Único de Saúde (SUS). Esse valor, que se refere às demandas judiciais para aquisição de medicamentos, ocupou, naquele ano, a 13a posição na relação de todas as demandas por fármacos solicitados por essa via (Xavier, 2017).

Nesse cenário, os objetivos do presente trabalho foram, com base em modelo econômico, estimar a relação de custo-efetividade incremental da abiraterona versus tratamento padrão para pacientes com CaPm resistente à castração e em uso prévio de quimioterapia e também apresentar as estimativas do impacto orçamentário da incorporação desse fármaco no SUS.

\section{Métodos}

\section{Tipo de estudo e intervenções em comparação}

Foi desenvolvida uma avaliação econômica do tipo custo-utilidade baseada em um modelo de Markov. As análises foram realizadas para o tratamento do câncer de próstata avançado, metastático, resistente à castração, comparando-se abiraterona (associada a corticoide) com tratamento padrão (apenas corticoide e melhor cuidado de suporte).

\section{Estrutura do modelo}

O modelo de Markov foi construído em MS Excel, baseado nas curvas de sobrevida global e sobrevida livre de progressão do PSA. Os estados de saúde do modelo foram paciente estável sem progressão, doença após progressão e óbito.

A população-alvo do modelo foi de homens com CaP metastático, resistentes à castração, que fizeram uso prévio de quimioterapia com docetaxel e, a despeito disso, tiveram progressão da doença ou toxicidade limitante.

O modelo foi desenvolvido com horizonte de tempo de cinco anos, compatível com a curta sobrevida dessa doença e tempo no qual a quase totalidade dos pacientes (99\%) foi a óbito na projeção modelada. 
A perspectiva considerada foi a do SUS, como fonte pagadora dos insumos e serviços em saúde, e a taxa de desconto, de $5 \%$ ao ano, foi aplicada a custos e efetividade (equivalente a 0,41\% ao mês).

\section{Estimativa dos parâmetros}

Para definir as estimativas de sobrevida global e sobrevida livre de progressão do PSA, foi conduzida uma revisão sistemática (RS) da literatura de acordo com as recomendações da Cochrane Collaboration (Higgins \& Green, 2011). Foram realizadas buscas por ensaios clínicos nas bases de dados do Medline (PubMed), Cochrane Central e Embase. Também foram revisadas as listas de referências dos estudos selecionados, não havendo, num primeiro momento, restrição por idioma nem data. Dois ensaios clínicos foram localizados, ambos tendo demonstrado mais efetividade da abiraterona quando comparada a placebo na população estudada (De Bono et al., 2011; Sun et al., 2016). Para o presente estudo, no entanto, utilizaram-se somente os parâmetros estimados no estudo COU-AA-301 (De Bono et al., 2011) devido à aplicabilidade para a população brasileira no que se refere ao contexto multicêntrico deste estudo e também ao perfil de pacientes de origem essencialmente ocidental apresentados.

Neste estudo, a sobrevida global dos pacientes que receberam abiraterona foi significantemente maior em relação àqueles que receberam placebo ( $H R=0,66$; IC95\%: 0,55-0,78), assim como a sobrevida livre de progressão do PSA (HR = 0,58; IC95\%: 0,46-0,73). No entanto, tais dados correspondiam a um tempo de seguimento inferior àquele necessário para aplicação no modelo econômico, sendo necessária a extrapolação de dados para o horizonte de tempo previamente definido.

A extração dos dados para extrapolação foi feita a partir de um estudo de custo-efetividade da abiraterona para o Reino Unido (NICE, 2016), o qual utilizou dados primários do ensaio clínico COU-AA-301 (De Bono et al., 2011) para estimar os dados de efetividade do medicamento. Nesse estudo de custo-efetividade, foram testados modelos paramétricos para extrapolação, elegendo-se a distribuição Weibull como aquela cujo modelo apresentou melhor ajuste aos dados. A extrapolação original, apresentada apenas no formato gráfiCo, foi digitalizada por meio do software Engauge Digitizer 9.8 e os dados foram exportados para tabela Excel. Para a abiraterona, foram digitalizados os dados das curvas de Weibull tanto para sobrevida global quanto para sobrevida livre de progressão. Para o placebo, foram usados os dados da curva de Weibull somente para sobrevida global, uma vez que não era necessário o uso de projeção para sobrevida livre de progressão, tendo esses dados sido observados até a progressão. A duração do ciclo escolhido foi de um mês, o qual é o mais frequentemente utilizado para doenças com rápida evolução, como o CaPm resistente à castração. Os valores obtidos são apresentados na Tabela 1.

Para definir os valores de utilidade, foi realizada uma busca na literatura. Nos portais Medline, SciELO e Lilacs, foram revisados estudos brasileiros que tivessem diretamente esti-

Tabela 1. Parâmetros utilizados no modelo

\begin{tabular}{|c|c|c|c|}
\hline Parâmetro & $\begin{array}{l}\text { Valor do } \\
\text { parâmetro }\end{array}$ & Limite & Fonte \\
\hline Hazard ratio/sobrevida global com abiraterona & 0,65 & & NICE, 2016 \\
\hline Hazard Ratio/sobrevida livre de progressão do PSA com abiraterona & 0,58 & & NICE, 2016 \\
\hline Utilidade pré-progressão & 0,7 & 0,86 & $\begin{array}{l}\text { Snedecor et al. 2013; } \\
\quad \text { Lloyd et al. } 2015\end{array}$ \\
\hline Utilidade pós-progressão & 0,5 & 0,32 & Sandblom et al. 2004 \\
\hline Ganho de utilidade com abiraterona & 0,046 & 0,069 & NICE, 2016 \\
\hline Utilidade morte & 0 & & \\
\hline Custo mensal abiraterona & $R \$ 6.844,00$ & $R \$ 3.422$ & CMED, SIASG \\
\hline Custo mensal prednisona & $\mathrm{R} \$ 6,00$ & & BPS \\
\hline Custo de cuidados paliativos em pacientes pós-progressão & $\mathrm{R} \$ 325,00$ & $\mathrm{R} \$ 975$ & \\
\hline Custo mensal médio com os exames* & $\mathrm{R} \$ 25,83$ & & SIGTAP \\
\hline Taxa de desconto mensal & $0,41 \%$ & $0 \%$ & \\
\hline
\end{tabular}

* Exames considerados na composição do valor: Tomografia computadorizada (não especificada qual região anatômica), a cada 6 semanas (portanto 0,67 ao mês), R\$ 138,67 (referência a tomografia de abdômen para determinação de custo); Ressonância magnética (não especificada qual região anatômica), a cada 6 semanas (portanto 0,67 ao mês), R\$ 268,75 (referência a ressonância de abdômen para determinação de custo); Eletrocardiograma, a cada 6 semanas (portanto 0,67 ao mês), $R \$ 5,15$; Ecografia (não especificada qual região anatômica), a cada 6 semanas (portanto 0,67 ao mês), R\$ 39,94 (usada a tomografia de abdômen para determinação de custo); Cintilografia óssea, a cada 6 semanas (portanto 0,67 ao mês), R\$ 190,99; Hemograma e PSA, a cada 6 semanas (portanto 0,67 ao mês), R\$ 21,57; Função renal, a cada 4 semanas, R\$ 3,70; Função hepática, a cada 2 semanas, R\$ 4,02. Todos os custos foram somados e ponderados pela periodicidade, considerando-se, ainda, que somente $5 \%$ dos pacientes fariam os exames (NICE). 
mado tais valores em pacientes com CaP avançado. Também se buscaram estudos que tivessem reportado uso de SF-36 ou EQ-5D, para posterior transformação em escores de utilidade. Não foram encontrados dados nacionais, sendo necessário recorrer a estimativas internacionais. Com base nas informações selecionadas, foram adotados os valores para doença pré-progressão, doença pós-progressão e ganho de utilidade com o uso da abiraterona em pacientes pré-progressão (Lloyd et al., 2015; NICE, 2016; Sandblom et al., 2004; Snedecor et al., 2013) (Tabela 1).

Não foram inseridos impactos de eventos adversos em custos e utilidade com o uso do medicamento, já que nos estudos consultados não houve diferenças em eventos adversos graus 3 e 4 (Fizazi et al., 2012; NICE, 2016).

\section{Uso de recursos e custos}

Para definição dos custos de exames para os pacientes pré-progressão, utilizaram-se os parâmetros de frequência de utilização de insumos de acordo com a literatura (NICE, 2016), convertendo-se os valores em reais (R\$) de acordo com o SIGTAP. Para definição do custo mensal médio, os valores de todos os exames foram somados e ponderados pela periodicidade. Para o modelo, considerou-se que apenas 5\% dos pacientes fariam os exames (NICE, 2016).

Assumiu-se que pacientes com doença sem progressão não sofreriam internações hospitalares e que para os pacientes pós-progressão os custos seriam oriundos dessas. Os dados de internação, entretanto, não foram localizados na literatura. Assim, para definição desse parâmetro, foi contatado o Serviço de Oncologia Clínica do Hospital Santa Rita (Porto Alegre, RS), tendo sido a estimativa de custo realizada com base em dados de 40 pacientes que foram a óbito por CaP metastático, considerando-se o número de internações e o tempo médio delas no período de oito meses antes do óbito. Essas definições estão de acordo com estudos internacionais, os quais apontam que grande parte dos custos com pacientes pós-progressão ocorre no período de seis a oito meses antes de sua morte (Krahn et al., 2010). Para atribuição dos custos a essas internações, foi feita pesquisa no DATASUS por valores de AlH para internações de cuidados a pacientes oncológicos.

O valor da abiraterona foi selecionado da Câmara de Regulação do Mercado de Medicamentos (CMED), consultando-se também os preços de compra no SIASG. Para a prednisona, foi considerada dose de $5 \mathrm{mg}$, duas vezes ao dia, em ambos os grupos, de acordo com o Banco de Preços em Saúde (BPS) referente a maio de 2017 (Tabela 1).

\section{Análise de sensibilidade}

Tendo em vista que os resultados de custo-efetividade foram desfavoráveis à abiraterona (ver abaixo na seção Resultados), a variação dos parâmetros foi feita apenas na direção do limite que poderia favorecer o medicamento, visto que a variação para o outro limite traria resultados mais extremos (isto é, que a abiraterona ficaria ainda menos custo-efetiva). Além disso, foram realizadas análises de limiar (threshold analysis), variando-se os parâmetros até que a razão de custo-efetividade incremental (RCEI) atingisse o valor de três vezes o PIB per capita. Esse valor sugerido pela Organização Mundial da Saúde é utilizado para um melhor julgamento do impacto nas análises de sensibilidade, sendo importante a utilização de algum valor limiar de referência, ainda que não haja tal valor explicitamente definido para o Brasil. Para a presente análise, foram variados os seguintes parâmetros: taxa de desconto, 0\% (valor extremo proposto na Diretriz de Avaliação Econômica do Ministério da Saúde) (Ribeiro et al., 2016); valores de utilidade pré-progressão e pós-progressão; ganho de utilidade com abiraterona; custo mensal do acompanhamento pós-progressão e cenário com as seis ocorrências anteriormente citadas de forma simultânea.

Também foi atribuído um cenário com uma taxa diferente de realização dos exames para avaliação de progressão. Para tal, considerou-se que os exames foram utilizados por todos os pacientes livres de progressão no grupo abiraterona, no mês 3 e a cada três meses depois disso (De Bono et al., 2011).

Considerando-se o mínimo impacto dos parâmetros de custo do corticoide e o custo mensal dos exames pré-progressão, não foi realizada análise de sensibilidade desses parâmetros. Também foi conduzida uma análise de limiar, variando a diferença de QALY que seria necessária para que a abiraterona se tornasse custo-efetiva, assumindo-se também o limiar de três vezes o PIB per capita.

\section{Impacto orçamentário}

Os principais parâmetros para a análise de impacto orçamentário foram o custo do medicamento, o tempo médio de tratamento e o tamanho da população-alvo. O custo do medicamento utilizado foi o do caso base, de R\$ 6.844 por mês.

Para o tempo de tratamento, foram encontrados dois valores diferentes, sendo necessário trabalhar com dois cenários. No primeiro, o valor foi oriundo do estudo COU-AA-301 com tempo médio de seguimento de 20,2 meses e tempo médio de tratamento de 7,4 meses (Fizazi et al., 2012). Esse valor, entretanto, é subestimado, pois neste momento ainda havia cerca de $25 \%$ de pacientes vivos, dentre os quais alguns ainda estavam em uso do fármaco. A outra estimativa é do modelo de Markov desenvolvido, em que o tempo médio dos pacientes no estado livre de progressão (em uso da abiraterona) foi de 12 meses. Considerando-se esse tempo, estipulou-se, então, que o medicamento seria utilizado apenas no primeiro ano (em todo ou em parte dele, considerando as duas fontes de dados) e que novas indicações nos anos 
subsequentes seriam pelo crescimento da população. Tendo em vista que (1) a condição clínica é extremamente grave, (2) a abiraterona tem poucos efeitos adversos e (3) que, se fosse incorporada, seria ao menos em um primeiro momento a única opção terapêutica nesses pacientes, adotou-se uma taxa de incorporação de 100\% desde o tempo zero.

A população-alvo foi estimada com base na análise dos bancos de APAC do DATASUS, com limpeza e combinação de todos os bancos em 2015 de todos os estados da federação. O número de pacientes únicos localizados no banco foi de 4.099, valor usado para cálculo do impacto orçamentário. Para novos casos, não foi considerado aumento da incidência, uma vez que os dados do INCA apontavam para 61.200 casos novos em 2016, tendo anteriormente apontado para 69 mil casos em 2014. Por outro lado, muitos casos prevalentes provavelmente progrediriam, passando a ser candidatos ao uso da abiraterona. Na ausência de dados fidedignos para essa estimativa, foram feitos três cenários, com 10\%, 20\% e $30 \%$ por ano (aplicados na estimativa de 4.099 casos no ano 1) de novos casos de câncer metastático, resistente à castração e com progressão após quimioterapia.

\section{Resultados}

Os resultados do caso base para um horizonte de cinco anos são apresentados na Tabela 2. O uso de abiraterona levou a um ganho de 0,310 ano de vida e 0,283 QALY, ajustada para taxa de desconto, com um custo adicional de R\$ 80.399, também com a aplicação da taxa de desconto. A custo-efetividade incremental do uso de abiraterona em relação ao tratamento usual foi de $\mathrm{R} \$ 284.416$ por QALY e de R\$ 259.319 por ano de vida ganho.

As análises de sensibilidade são apresentadas na Tabela 3. Em nenhum dos cenários variando cada parâmetro isoladamente, assim como no cenário de variação simultânea dos seis parâmetros para os seus valores mais favoráveis à abiraterona, foi atingida RCEI abaixo de três vezes o PIB per capita (que foi de R\$31.587 em 2017). Considerando que nem nessa situação de variação simultânea de todos os parâmetros para o valor mais favorável à abiraterona foi atingido um valor abaixo de um limiar arbitrário de três vezes o PIB per capita, foi julgado desnecessário realizar uma análise probabilística, pois inequivocamente o resultado seria de 100\% das simulações acima de tal limiar.

Na análise do cenário de realização de exames em todos os pacientes livres de progressão no grupo abiraterona, os resultados ficaram mais desfavoráveis: R\$ 289.305 por QALY e $R \$ 263.777$ por AVG.

Nas análises de limiar, nem considerando o valor mínimo da taxa de desconto, o valor máximo de utilidade pré-progressão, o valor mínimo da utilidade pós-progressão, o valor máximo para ganho de utilidade com abiraterona, o medica-

Tabela 2. Resultados do caso base

\begin{tabular}{lccc}
\hline Parâmetro & Abiraterona & Tratamento padrão & Incremental \\
\hline QALY total (sem desconto) & 1,104 & 0,797 & 0,307 \\
\hline QALY total (com desconto) & 1,045 & 0,763 & 0,283 \\
\hline Anos de vida ganhos (sem desconto) & 1,705 & 1,361 & 0,344 \\
\hline Anos de vida ganhos (com desconto) & 1,609 & 1,299 & 0,310 \\
\hline Custo total (sem desconto) & $\mathrm{R} \$ 86.834,29$ & $\mathrm{R}$ 3.087,12 & $\mathrm{R} \$ 83.747,17$ \\
\hline Custo total (com desconto) & $\mathrm{R} \$ 83.295,02$ & $\mathrm{R} 2.895,32$ & $\mathrm{R} \$ 80.399,70$ \\
\hline RCEl: R\$/QALY (com desconto) & - & - & $\mathrm{R} \$ 284.416,67$ \\
\hline RCEI: R\$/AVG (com desconto) & - & - & $R \$ 259.319,95$ \\
\hline
\end{tabular}

Tabela 3. Análises de sensibilidade determinísticas

\begin{tabular}{lccc}
\hline Parâmetro & Valor do parâmetro & Valor da RCEI (QALY) & Valor da RCEI (AVG) \\
\hline Taxa de desconto & $0 \%$ & $\mathrm{R} \$ 272.667,327$ & $\mathrm{R} \$ 243.107,140$ \\
\hline Valor de utilidade pré-progressão & 0,86 & $\mathrm{R} \$ 230.532,302$ & - \\
\hline Valor de utilidade pós-progressão & 0,32 & $\mathrm{R} \$ 266.923,553$ & - \\
\hline Ganho de utilidade com abiraterona & 0,069 & $\mathrm{R} \$ 263.417$ & $\mathrm{R}$ \\
\hline Custo mensal da abiraterona & $\mathrm{R} \$ 3.422$ & $\mathrm{R} \$ 142.088,120$ & $\mathrm{R} \$ 129.550,365$ \\
\hline Custo mensal do acompanhamento pós-progressão & $\mathrm{R} \$ 975$ & $\mathrm{R} \$ 281.576,781$ & $\mathrm{R} \$ 256.730,646$ \\
\hline $\begin{array}{l}\text { Cenário com as seis ocorrências anteriormente } \\
\text { citadas de forma simultânea }\end{array}$ & - & $\mathrm{R} \$ 98.330,855$ & $\mathrm{R} \$ 119.358,418$ \\
\hline
\end{tabular}


mento tornar-se-ia custo-efetivo, tanto considerando o limiar de três vezes o PIB per capita para QALY ganho como ano de vida ganho. Para que o limiar de três vezes o PIB per capita por QALY fosse atingido, o custo mensal pós-progressão precisaria chegar a $\mathrm{R} \$ 45.000$ (e, para alcançar esse limiar em termos de R\$ por ano de vida ganho, R\$ 43.000 mensais). Para que o limiar de três vezes o PIB per capita fosse atingido nos desfechos de R\$ por QALY e R\$ por ano de vida ganho, o custo mensal da abiraterona não poderia ser superior a R\$2.100 e $R \$ 2.400$, respectivamente. Finalmente, fizemos uma análise de limiar alterando diretamente o desfecho: seria necessário que o valor fosse de 0,90 (tanto para QALY como anos de vida ganhos) para que a abiraterona ficasse abaixo do limiar de três vezes o PIB per capita.

Os resultados da análise de impacto orçamentário podem ser vistos na Tabela 4. No cenário mais conservador, com 7,4 meses de tempo médio de tratamento e 10\% de novos casos por ano, o impacto seria de $\mathrm{R} \$ 270$ milhões em cinco anos. No cenário mais arrojado, com média de 12 meses de tratamento e $30 \%$ de novos casos por ano, os valores seriam de $\mathrm{R} \$ 740$ milhões em cinco anos.

\section{Discussão}

A revisão sistemática da literatura demonstra que a abiraterona é mais efetiva que o placebo em pacientes com CaP resistente à castração, após progressão da doença. O benefí- cio em termos absolutos, porém, é de aumento de sobrevida global inferior a quatro meses. A análise de custo-efetividade demonstrou uma relação incremental alta, acima de $\mathrm{R} \$ 250$ mil, tanto para o desfecho de QALY como de anos de vida ganhos. A análise de sensibilidade mostrou que, mesmo considerando todos os parâmetros mais importantes do modelo em seu extremo mais favorável à abiraterona, essa relação de custo-efetividade ainda permaneceria acima do triplo do PIB per capita brasileiro. No impacto orçamentário, as cifras são elevadas, com impacto de R\$290 milhões em cinco anos, mesmo no cenário com parâmetros mais conservadores.

No nosso estudo, as curvas do modelo foram definidas com base na extrapolação de dados do principal estudo com o medicamento, o qual apresentou uma razão de risco (HR) de 0,65, IC95\%: 0,54 a 0,77 (De Bono et al., 2011). Considerando-se o intervalo de confiança para o cenário mais favorável, ou seja, HR para sobrevida global de 0,54 (menos de 20\% meIhor do que o caso base), verificamos que é inverossímil que seja possível que o ganho tanto em QALY quanto em ano de vida ganho triplique (uma vez que os resultados do caso base foram de 0,30 e seria necessário que chegassem a 0,90 para que se alcançasse um resultado abaixo de três vezes o PIB per capita).

Dados sobre ganho de QALY e anos de vida em outros modelos de custo-efetividade da abiraterona estão disponíveis na literatura internacional. Os resultados desses estudos em termos de QALY e anos de vida ganhos (abiraterona ver-

Tabela 4. Análise de impacto orçamentário

\begin{tabular}{|c|c|c|c|c|c|c|}
\hline Cenário & Ano 1 & Ano 2 & Ano 3 & Ano 4 & Ano 5 & Total \\
\hline \multicolumn{7}{|c|}{ Tempo de tratamento de 12 meses; $10 \%$ de casos novos nos anos subsequentes } \\
\hline Tamanho da população-alvo & 4.099 & 410 & 410 & 410 & 410 & - \\
\hline Custo total & $R \$ 336.642 .672$ & $R \$ 33.672 .480$ & $\mathrm{R} \$ 33.672 .480$ & $\mathrm{R} \$ 33.672 .480$ & $R \$ 33.672 .480$ & $\mathrm{R} \$ 471.332 .592$ \\
\hline \multicolumn{7}{|c|}{ Tempo de tratamento de 12 meses; $20 \%$ de casos novos nos anos subsequentes } \\
\hline Tamanho da população-alvo & 4.099 & 820 & 820 & 820 & 820 & - \\
\hline Custo total & $R \$ 336.642 .672$ & $R \$ 67.344 .960$ & $R \$ 67.344 .960$ & $R \$ 67.344 .960$ & $\mathrm{R} \$ 67.344 .960$ & $R \$ 606.022 .512$ \\
\hline \multicolumn{7}{|c|}{ Tempo de tratamento de 12 meses; $30 \%$ de casos novos nos anos subsequentes } \\
\hline Tamanho da população-alvo & 4.099 & 1.230 & 1.230 & 1.230 & 1.230 & - \\
\hline Custo total & $R \$ 336.642 .672$ & $R \$ 101.017 .440$ & $\mathrm{R} \$ 101.017 .440$ & $\mathrm{R} \$ 101.017 .440$ & $R \$ 101.017 .440$ & $R \$ 740.712 .432$ \\
\hline \multicolumn{7}{|c|}{ Tempo de tratamento de 7,4 meses; $10 \%$ de casos novos nos anos subsequentes } \\
\hline Tamanho da população-alvo & 4.099 & 410 & 410 & 410 & 410 & - \\
\hline Custo total & R\$ 207.596 .314 & $\mathrm{R} \$ 20.764 .696$ & $\mathrm{R} \$ 20.764 .696$ & $\mathrm{R} \$ 20.764 .696$ & $R \$ 20.764 .696$ & $\mathrm{R} \$ 290.655 .098$ \\
\hline \multicolumn{7}{|c|}{ Tempo de tratamento de 7,4 meses; $20 \%$ de casos novos nos anos subsequentes } \\
\hline Tamanho da população-alvo & 4.099 & 820 & 820 & 820 & 820 & - \\
\hline Custo total & $\mathrm{R} \$ 207.596 .314$ & $R \$ 41.529 .392$ & $R \$ 41.529 .392$ & $R \$ 41.529 .392$ & $R \$ 41.529 .392$ & $\mathrm{R} \$ 373.713 .882$ \\
\hline \multicolumn{7}{|c|}{ Tempo de tratamento de 7,4 meses; $30 \%$ de casos novos nos anos subsequentes } \\
\hline Tamanho da população-alvo & 4.099 & 1.230 & 1.230 & 1.230 & 1.230 & \\
\hline Custo total & $\mathrm{R} \$ 207.596 .314$ & $R \$ 62.294 .088$ & $R \$ 62.294 .088$ & $R \$ 62.294 .088$ & $R \$ 62.294 .088$ & $R \$ 456.772 .666$ \\
\hline
\end{tabular}


sus tratamento padrão) variam amplamente, havendo dois estudos cujos resultados de 0,06 e 0,27 foram menos favoráveis que o do presente trabalho (Pereira et al., 2012; Zhong et al., 2013); um estudo chegou a 0,30, número quase idêntico ao aqui estimado (Chopra et al., 2012); e dois trabalhos encontraram valores de 0,47 (Marin Piva et al., 2016; Persson et al., 2012), maiores do que os apresentados na presente avaliação. Porém, conhecendo-se o custo do medicamento no Brasil, mesmo que tivéssemos encontrado os valores mais favoráveis da literatura (Marin Piva et al., 2016), os resultados para um limiar de três vezes o PIB per capita ainda não seriam custo-efetivos.

No que diz respeito ao impacto orçamentário, comparando-se o custo da abiraterona com o de outros medicamentos recentemente incorporados utilizados para o tratamento do câncer, não há como assumir um cenário favorável para esse medicamento. No nosso estudo, adotamos uma taxa de incorporação de $100 \%$ no tempo zero, com incerteza de tempo de tratamento que poderia variar de 7 a 12 meses. Nesse sentido, mesmo que assumíssemos um tempo de tratamento de sete meses, o qual teria um impacto mínimo, este seria de $R \$$ 290 milhões. Para a incorporação do medicamento pertuzumabe, por exemplo, incorporado em 2017 para o tratamento do câncer de mama HER2-positivo metastático em primeira linha de tratamento associado a trastuzumabe e docetaxel, embora no parecer o medicamento não tenha se mostrado custo-efetivo, o cenário alternativo apresentado pelo fabricante demonstrou um impacto orçamentário de 201 milhões de reais em cinco anos (Ministério da Saúde, 2017), ou seja, quase 90 milhões a menos do impacto avaliado para incorporação da abiraterona.

No que diz respeito ao cenário internacional, faz-se interessante analisar a perspectiva de países como Inglaterra e Canadá. Na Inglaterra, a abiraterona foi disponibilizada como opção para pacientes com as condições clínicas da população-alvo do presente estudo, desde que houvesse progressão documentada após uso de docetaxel e o preço fosse mantido conforme acordo comercial com o National Health System da Inglaterra (NHS) da Inglaterra. Nesse país, a RCEI de submissão foi de $£ 46,800$ por QALY, tendo o NICE considerado que o valor real seria maior, mas não superior a $£ 50,000$ por QALY.

No Canadá, o Pan-Canadian Oncology Drug Review (pCODR) recomendou a incorporação do medicamento em 2013, mas somente para pacientes assintomáticos ou levemente sintomáticos resistentes à terapia antiandrogênica que não tivessem recebido quimioterapia prévia e com ECOG entre 0 e 1. A RCEl apresentada na submissão foi de C $\$ 128.197$ por QALY, o qual poderia chegar a $C \$ 258,428$ por QALY, de acordo com o comitê de avaliação. Assim, realizando-se a conversão para a moeda brasileira, tanto do valor na Inglaterra quanto aquele apresentado no Canadá, pode-se inferir que no Brasil as estimativas estariam mais próximas do cenário europeu do que em relação ao canadense, sendo necessário considerar o cenário econômico de cada um dos países para subsidiar uma tomada de decisão.

É importante reconhecer algumas limitações do nosso estudo. Primeiro, não foi possível variar os parâmetros de efetividade em sobrevida global e livre de progressão, tendo em vista o tipo de modelo construído. Além disso, foi necessário utilizar dados de utilidade internacionais, uma vez que dados nacionais não estavam disponíveis. Outra limitação diz respeito aos dados de custo de seguimento pós-progressão, os quais são oriundos de uma pequena amostra, da cidade de Porto Alegre, sendo feita uma suposição sobre o momento de progressão (foram considerados os custos nos oito meses anteriores ao óbito). Contudo, considerando a robustez dos resultados em relação à incerteza desses parâmetros, tais limitações não parecem ter impacto nas conclusões do estudo.

\section{Conclusões}

Estudos demonstram que a abiraterona é efetiva para o tratamento do $\mathrm{CaPm}$ resistente à castração em pacientes com uso prévio de quimioterapia, quando comparada ao uso de placebo. No entanto, os ganhos de sobrevida, tanto global quanto livre de progressão do PSA, são pequenos e o elevado custo do tratamento torna-o não custo-efetivo mesmo se considerando todas as incertezas do modelo de análise favoráveis ao medicamento e também um limiar elevado, de três vezes o PIB per capita. No nosso estudo, a RCEl atingiu cerca de nove vezes o PIB per capita por QALY, com um cenário de impacto financeiro importante sobre o SUS caso o medicamento fosse incorporado.

\section{Agradecimentos}

Agradecemos à equipe técnica da Conitec, em especial ao analista de Políticas Sociais do Ministério da Saúde Ivan Ricardo Zimmermann, pelos esclarecimentos referentes à extração primária de dados do DATASUS e também a questões relacionadas a custos de medicamentos.

\section{Referências bibliográficas}

Chopra A, Gluck S, Montero AJ, Kumar K, Avancha VR, Lopes G. Costeffectiveness evaluation of abiraterone in the treatment of patients with castration-resistant prostate cancer who previously received docetaxel. J Clin Oncol. 2012;30.

Cornford P, Bellmunt J, Bolla M, Briers E, De Santis M, Gross T, et al. EAUESTRO-SIOG guidelines on prostate cancer. Part II: treatment of relapsing, metastatic, and castration-resistant prostate cancer. Eur Urol. 2017;71:630-42. doi:10.1016/j.eururo.2016.08.002.

De Bono JS, Logothetis CJ, Molina A, Fizazi K, North S, Chu L, et al. Abiraterone and increased survival in metastatic prostate cancer. N Engl J Med. 2011;364:1995-2005. 
De Bono JS, Oudard S, Ozguroglu M, Hansen S, Machiels J-P, Kocak I, et al. Prednisone plus cabazitaxel or mitoxantrone for metastatic castrationresistant prostate cancer progressing after docetaxel treatment: a randomised open-label trial. Lancet. 2010;376:1147-54. doi:10.1016/S01406736(10)61389-X.

Fizazi K, Scher HI, Molina A, Logothetis CJ, Chi KN, Jones RJ, et al. Abiraterone acetate for treatment of metastatic castration-resistant prostate cancer: final overall survival analysis of the COU-AA-301 randomised, doubleblind, placebo-controlled phase 3 study. Lancet Oncol. 2012;13:983-92. doi:10.1016/S1470-2045(12)70379-0.

Hellerstedt BA, Pienta KJ. The current state of hormonal therapy for prostate cancer. CA Cancer J Clin. 2002;52:154-79.

Higgins JPT, Green S (editors). Cochrane handbook for systematic reviews of interventions version 5.1.0 2011.

Inca. Atlas de Mortalidade por Câncer 1996-2014. 2014.

Inca. Estimativa 2018: incidência de câncer no Brasil/Instituto Nacional de Câncer José Alencar Gomes da Silva; 2017.

Krahn MD, Zagorski B, Laporte A, Alibhai SMH, Bremner KE, Tomlinson G, et al. Healthcare costs associated with prostate cancer: estimates from a population-based study. BJU Int. 2010;105:338-46. doi:10.1111/j.1464410X.2009.08758.x.

Lloyd AJ, Kerr C, Penton J, Knerer G. Health-related quality of life and health utilities in metastatic castrate-resistant prostate cancer: a survey capturing experiences from a diverse sample of UK patients. Value Health. 2015;18:1152-7. doi:10.1016/j.jval.2015.08.012.

Logothetis CJ, Basch E, Molina A, Fizazi K, North SA, Chi KN, et al. Effect of abiraterone acetate and prednisone compared with placebo and prednisone on pain control and skeletal-related events in patients with metastatic castration-resistant prostate cancer: exploratory analysis of data from the COU-AA-301 randomised trial. Lancet Oncol. 2012;13:12107. doi:10.1016/S1470-2045(12)70473-4.

Marin Piva H, Castro Cordero JA, Sabater Cabrera E. Cost-effectiveness analysis of abiraterone in patients with metastatic, castration-resistant, prostate cancer with progression after receiving chemotherapy with docetaxel, compared with receiving only palliative support: the perspective of the Costa Rican public health system (Caja Costarricense De Seguro Social). Value Health. 2016;19:A154.

Ministério da Saúde. Pertuzumabe para o tratamento do câncer de mama HER2-positivo metastático em primeira linha de tratamento associado ao trastuzumabe e docetaxel. 2017.

NICE. Technology appraisal guidance. Abiraterone for treating metastatic hormone-relapsed prostate cancer before chemotherapy is indicated. 2016.
Pereira ML, Bahmdouni LSK, Pepe C, Teich V. Cost-effectiveness analysis of abiraterone for the treatment of advanced prostate cancer under the Brazilian private health care system. Value Health. 2012;15:A424-5

Persson U, Nilsson S, Hjortsberg C, Prûtz C. Economic evaluation of abiraterone acetate as treatment for metastatic castration resistant prostate cancer after failure of docetaxel in Sweden. Value Health. 2012;15:A219. doi:10.1016/j.jval.2012.03.1183.

Ribeiro RA, Neyeloff JL, Itria A, Santos VCC, Vianna CMM, Silva EN, et al. Diretriz metodológica para estudos de avaliação econômica de tecnologias em saúde no Brasil. J Bras Econ Saúde. 2016;8(3):174-84.

Sandblom G, Carlsson P, Sennfalt K, Varenhorst E. A population-based study of pain and quality of life during the year before death in men with prostate cancer. Br J Cancer. 2004;90:1163-8. doi:10.1038/sj.bjc.6601654.

Scher HI, Fizazi K, Saad F, Taplin M-E, Sternberg CN, Miller K, et al. Increased survival with enzalutamide in prostate cancer after chemotherapy. N Engl J Med. 2012;367:1187-97. doi:10.1056/NEJMoa1207506.

Siegel R, Naishadham D, Jemal A. Cancer statistics, 2013. CA Cancer J Clin. 2013;63:11-30. doi:10.3322/caac.21166.

Snedecor SJ, Carter JA, Kaura S, Botteman MF. Denosumab versus zoledronic acid for treatment of bone metastases in men with castration-resistant prostate cancer: a cost-effectiveness analysis. J Med Econ. 2013;16:19-29. doi:10.3111/13696998.2012.719054.

Sun Y, Zou Q, Sun Z, Li C, Du C, Chen Z, et al. Abiraterone acetate for metastatic castration-resistant prostate cancer after docetaxel failure: a randomized, double-blind, placebo-controlled phase 3 bridging study. Int J Urol. 2016;23:404-11.

Tannock IF, de Wit R, Berry WR, Horti J, Pluzanska A, Chi KN, et al. Docetaxel plus prednisone or mitoxantrone plus prednisone for advanced prostate cancer. N Engl J Med. 2004;351:1502-12. doi:10.1056/NEJMoa040720.

Van Praet C, Rottey S, Van Hende F, Pelgrims G, Demey W, Van Aelst F, et al. Abiraterone acetate post-docetaxel for metastatic castrationresistant prostate cancer in the Belgian compassionate use program. Urol Oncol Semin Orig Investig. 2016;34:254.e7-254.e13. doi:10.1016/j. urolonc.2015.12.017.

Xavier CA. Judicialização da Saúde no Brasil: principais projetos desenvolvidos pela coordenação de assuntos judiciais. Brasília; 2017.

Zhong L, Pon V, Srinivas S, Nguyen N, Frear M, Kwon S, et al. Therapeutic options in docetaxel-refractory metastatic castration-resistant prostate cancer: a cost-effectiveness analysis. PLoS One. 2013;8:e64275. doi:10.1371/journal.pone.0064275. 